\title{
Neural Network analysis of Fractal Koch Antenna
}

\author{
Varindra Kumar \\ Dept. of Aeronautics Engineering \\ Imperial College London \\ London, UK \\ Varindra@ieee.org
}

\begin{abstract}
The paper proposes an economical, small size and compact fractal Koch dipole antenna for its resonance within $4-5 \mathrm{GHz}$ frequency band. The antenna and its array have been bent to calculate and show the parametric behavior with its curvature. The reflection parameter and its gain within the frequency band has also been calculated and compared with open end, short end and its bend configuration. A trained neural network function has been used to calculate antenna parameters of the array antenna using the known parameters of single element antenna. In addition the paper also provides frequency dependent impedance plot using ADS tool and its matching circuit for its application within controlled impedance PCB environment.
\end{abstract}

Keywords-Koch Antenna, Dipole, Array, S11 parameter, Neural Network

\section{INTRODUCTION}

Miniaturized wireless devices have pushed the demand for small and compact subcomponents including the communicating devices with antenna. In addition to the reduction in the antenna size, the operating bandwidth needs to be wider to transmit and receive the signal without any loss and at the same time it also needs to have multifrequency resonance. An optimized fractal antenna due to its space filling nature can provide higher bandwidth and multi frequency resonance in addition to providing an excellent gain and radiation efficiency. Further the structure can also be tuned to other frequency with small alteration in the metallic connectivity within it. The fractal antenna also takes less dimensional space with its length and width being smaller than the other similar type of antennas. Various literatures have described fractal geometry such as Koch, Sierpinski, Hilbert, Minkowski, Peano, Euclidean [1 - 4] based antenna but these mainly have been designed as monopole and few have been designed as dipole antenna [5 7]. In addition, a suitable wire thickness or trace width of the design can provide better impedance matching for its integration with other PCB interconnects and components. The paper here has been organised with the design methodology to describe the mechanism to generate the Koch fractal shape with open, short and bend structures while the modeling and simulation section talks about the geometry of the structure. The subsection also describes the neural network mechanism to train the antenna parameters. A comparison result for these structures for various antenna parameters such as reflection, transmission, gain and impedance over its frequency range with the feed forward neural network trained parametric result for the antenna array has also been obtained here. Finally conclusion has been described.

\section{Design Methodology}

The dipole antenna with a fractal complexity within the design provides additional dimensional space and cost saving for its design with using a simple wire in crude form or a metallic trace and without any other substrate or media. The dipole Koch fractal antenna has been designed using a Matlab script using (1) and is imported with its vector representation in CST tool. Here ' $\mathrm{s}$ ' is the length of the segment at stage ' $\mathrm{m}$ ' while the number of segment is $\mathrm{N}(\mathrm{s})$, thus the total length at stage ' $m$ ' is provided by (2). Finally the divider dimension of the segment can be obtained by (3) [8 - 10]. The antenna with its open end using the Koch fractal design is shown in Fig. 1. After open end design, the short end configuration has been created using extrude and loft feature present in the CST tool and is shown in Fig. 2. Fig. 2 is also used to show the short metallic effect of its structure over its frequency range. In addition the antenna is bent over a cylindrical surface to show the curvature effect of its behavior for various antenna parameters such as reflection, gain, directivity etc. and is shown in Fig. 3. These parameters can be obtained with the traditional transmission line theorem when the structure is properly meshed and solved in either frequency or time domain using various well established meshing and modeling methods. The reflection parameter can be defined using (4) where $Z_{\text {in }}$ is the structure impedance and $Z_{c}$ is the characteristic impedance, however it has been obtained here with the field solver tool present in the CST software. The differential impedance in the case of dipole antenna can be obtained with the differential value of the single ended impedance calculation. The $Z_{\text {in }}$ can also be obtained using (5) with its reflection (S11) and transmission (S12) parameters. The bandwidth of the resonating structure can be obtained using (4) where the $f_{H}$ and $f_{L}$ represent the highest and lowest frequency of the $90 \%$ minimum reflection coefficient, subject to value less than $-10 \mathrm{~dB}$.

$$
s=\left(\frac{1}{3^{m}}\right) \text { and } N(s)=4^{m}
$$




$$
\begin{gathered}
\text { Length }_{\text {total }}=4^{m}\left(\frac{1}{3^{m}}\right) \\
D=\frac{\log 4}{\log (1 / s)} \\
S_{11}=\frac{Z_{\text {in }}-Z_{c}}{Z_{\text {in }}+Z_{c}} \\
Z_{\text {in }}=\sqrt{\frac{\left(1+S_{11}\right)^{2}-S_{21}^{2}}{\left(1-S_{11}\right)^{2}-S_{21}^{2}}} \\
B W(\%)=\frac{f_{H}-f_{L}}{f_{c}} \times 100 \\
7.85 \mathrm{~mm}
\end{gathered}
$$

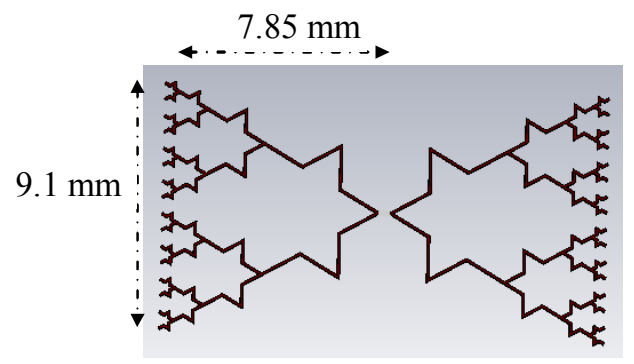

Fig. 1. Fractal antenna - open configuration

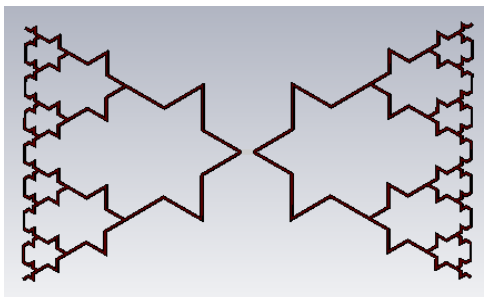

Fig. 2. Fractal antenna - short configuration

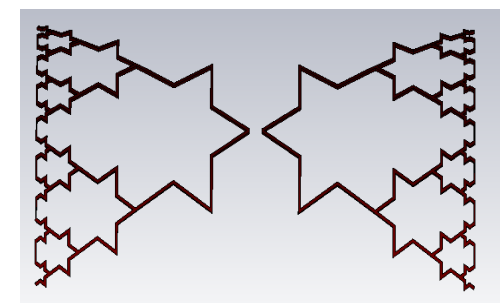

Fig. 3. Fractal antenna short - bend configuration

\section{Modeling And Simulation Results}

The fractal Koch antenna shown in Figs $1-3$ has been designed using CST software and is simulated with its transient solver tool. The antenna has been configured in dipole mode with a discrete port exciting the port. Once the open end structure is modeled, the outside edge is shorted to create a short end configuration. The structure is bent over 7 $\mathrm{mm}$ radius air cylinder to calculate the antenna parameters with its bending effect. Similarly the array is formed with 0.5 $\mathrm{mm}$ spacing of parallel dipole antennas.

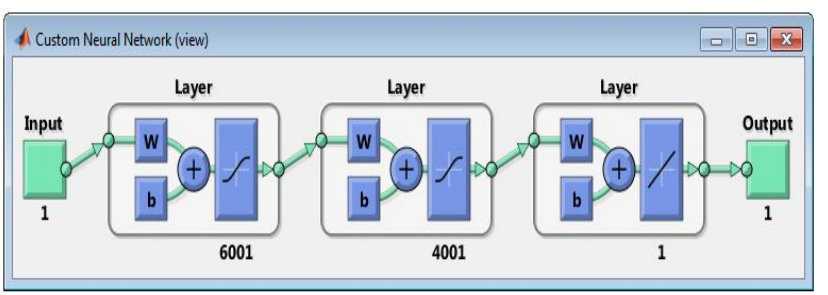

Fig. 4. Neural Network configuration

A neural network with various layers can be a perfect mechanism to obtain the output from the given input and once the network is properly trained with some functions, it can provide the desired output. A feed-forward network with multilayer perceptron has been designed to obtain the gain for the array antenna using the gain of $\mathrm{x} 1$ antenna as its input. Instead of using the neural network tool present in the Matlab, a script has been written here. The classic gradient descent function 'traingd' with trainlm 'Lavenberg-

(6) Marquardt' training function has been used to train the system [11 - 12]. The gain for $\mathrm{x} 1$ antenna is fed as its input and passed through three layers of network with layer one and two as hidden layers consisting of 6001 and 4001 neurons and defined by tansig function while the layer three consists of one number of neuron with a linear function. Functions tansig and linear can be defined using $(7-8)$ and its plot is shown in Fig. 5. The learning rate and the parametric goal of the neural network training are defined as $1 \mathrm{e}-2$ and $1 \mathrm{e}-3$.

$$
\tan \operatorname{sig}(x)=\frac{2}{[1+\exp (-2 x)]}
$$

$$
\operatorname{purelin}(x)=x
$$

\section{tansig(x) function purelin(x) function}
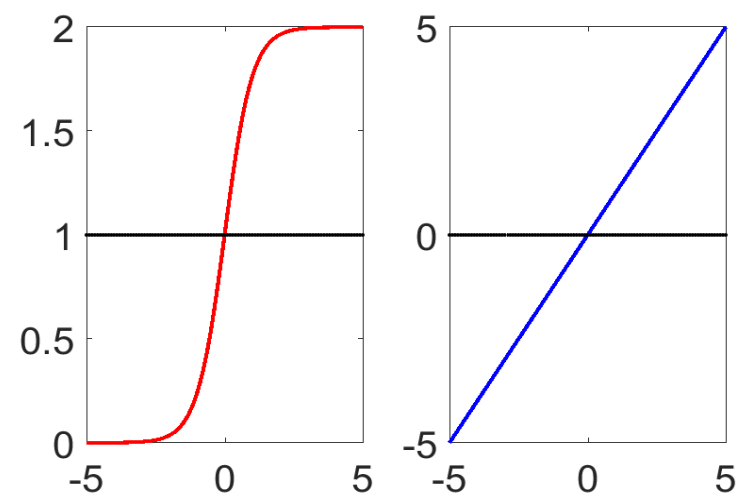

Fig. 5. Tansig and purelin function used in the neural network

\section{A. Simulation results for the fractal Koch $x 1$ antenna}

After simulating the block in time domain, the S11 and gain parameters for the open, short and short - bend fractal antenna configurations have been obtained and are shown in Figs 6 and 7. The S11 parameter can also be obtained using (2) with creating an efficient mesh of the block while the gain can be calculated using (3). The S11 parametric comparison shows that the open and short end fractal antenna resonates at nearly the same resonance frequency with 5.31 and $5.28 \mathrm{GHz}$ with $-15.2 \mathrm{~dB}$ reflection value while the short end - bend configuration resonates at $4.86 \mathrm{GHz}$ 
with $-12.6 \mathrm{~dB}$ reflection value. Similarly Fig. 7 represents the comparison of the farfield gain with nearly $2.05 \mathrm{~dB}$ for open and short end configuration while the parametric bend configuration shows a gain of $1.92 \mathrm{~dB}$. The bandwidth for open, short and short - bend configuration is calculated to be $0.69 \mathrm{GHz}, 0.7 \mathrm{GHz}$ and $0.45 \mathrm{GHz}$.

\section{Comparison of $\mathbf{S 1 1}$ parameter - x1 antenna}

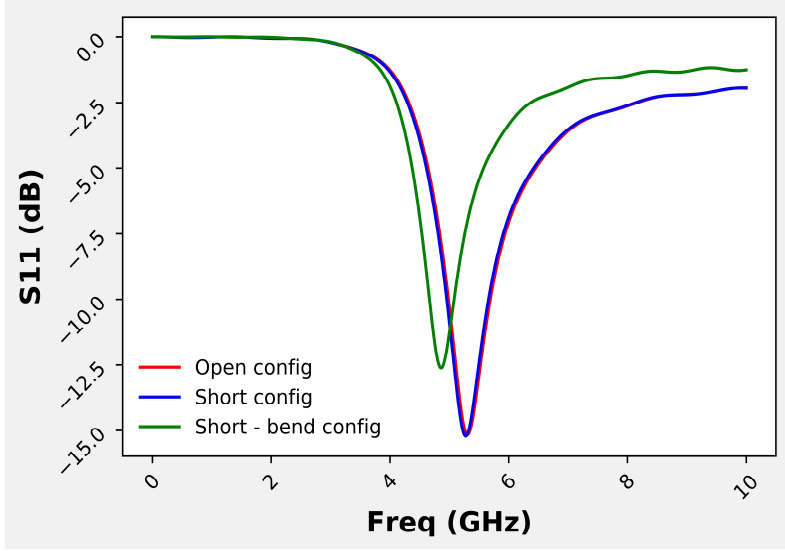

Fig. 6. Comparison of $\mathrm{S} 11$ parameter for $\mathrm{x} 1$ fractal antenna

\section{Comparison of gain - x1 antenna}

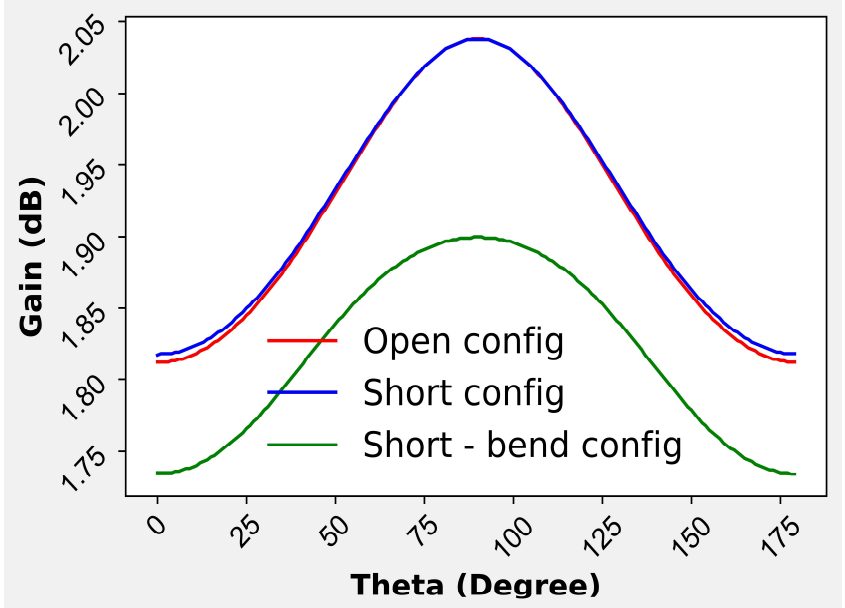

Fig. 7. Comparison of farfield gain for $\mathrm{x} 1$ fractal antenna

The S-parameters with touchstone file has been imported within the ADS tool and converted to the magnitude and phase of its impedance. The frequency dependent impedance and phase plot of these three types of the configuration is obtained to integrate the design within an impedance controlled PCB environment and is shown in Fig. 8. The solid line presents the impedance behavior while the dashed line represents the phase behavior. The electronic designs within the PCB are impedance controlled with 50 or $75 \mathrm{ohm}$ for single ended circuit and $100 \mathrm{ohm}$ for the differential ended circuit for an efficient power transfer, hence an impedance matching circuit has been designed using the smith chart component and is shown in Fig. 9. The parametric values of $L_{s}$ and $C_{s}$ to match the antenna load impedance to $50 \mathrm{ohm}$ source impedance has been obtained for these configurations and is provided in the Table I. Thus a simple tuning circuit with tune values can be used to match the input impedance of the antenna to the trace impedance within the PCB environment.

\section{Comparison of Impedance - $x \mathbf{1}$ antenna}

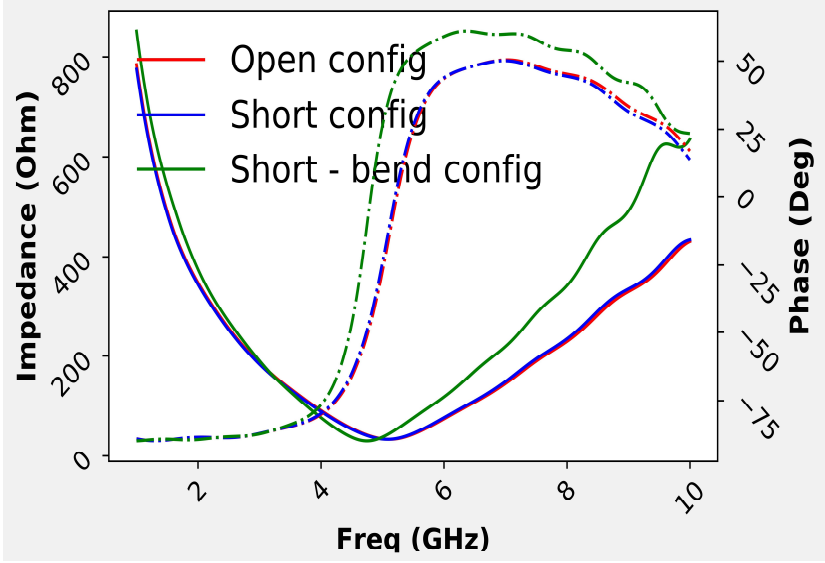

Fig. 8. Comparison of impedance for $\mathrm{x} 1$ fractal antenna

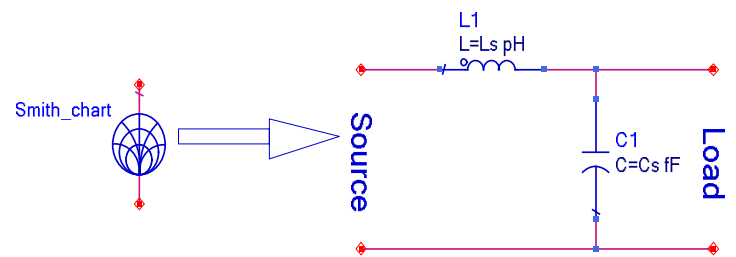

Fig. 9. Impedance matching circuit for fractal antenna

TABLE I. LC parameters for Impedance matching

\begin{tabular}{|l|c|c|c|}
\hline & Open config & Short config & Short - bend config \\
\hline $\mathrm{L}_{\mathrm{s}}(\mathrm{pH})$ & 487.24485 & 488.2616 & 653.53321 \\
\hline $\mathrm{C}_{\mathrm{s}}(\mathrm{fF})$ & 460.09507 & 468.19573 & 555.15063 \\
\hline
\end{tabular}

\section{B. Simulation results for the fractal Koch array antenna}

Here an array of the koch antenna with two dipole antenna placed at a vertical spacing of $0.5 \mathrm{~mm}$ has been designed and excited for the calculation of S11 and gain parameters. Similar to the configuration of the $\mathrm{x} 1$ antenna, three different configurations such as open end, short end and short end with bend have been designed and are shown in Figs. $10-12$. The short end configuration is bent over an air cylinder with radius $7 \mathrm{~mm}$ to create short end - bend configuration.

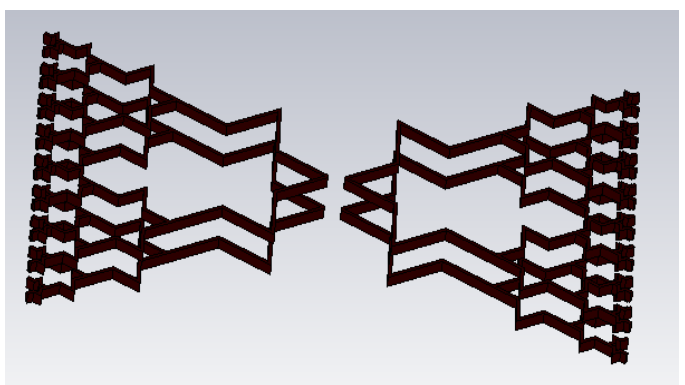

Fig. 10. Fractal Array antenna - open configuration

The reflection parameter obtained in Fig. 13 shows that the open and short end array antenna resonates at nearly the same resonance frequency of $4.72 \mathrm{GHz}$ and $4.65 \mathrm{GHz}$ with $12.3 \mathrm{~dB}$ and $-12.8 \mathrm{~dB}$ S11 value while the short end - bend configuration resonates at $4.0 \mathrm{GHz}$ with $-20 \mathrm{~dB}$ S11 value. The bandwidth for open end array, short end array and short end - bend array is calculated and found to be $0.72 \mathrm{GHz}$, 
$0.78 \mathrm{GHz}$ and $0.89 \mathrm{GHz}$. To show the parametric behavior, the gain and resonance frequency of the dipole antenna with its bending of radius from $3 \mathrm{~mm}$ to $9 \mathrm{~mm}$ for the fractal short - bend and fractal short - bend array configuration is also obtained and plotted in Fig. 17. Table II shows the LC parameters for matching the impedance to $50 \Omega$ for the array configuration.

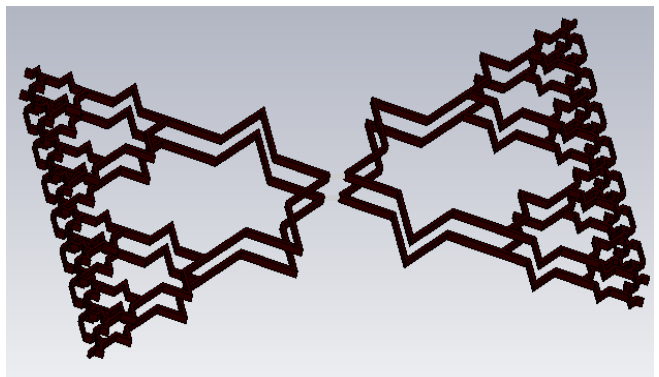

Fig. 11. Fractal array antenna - short configuration

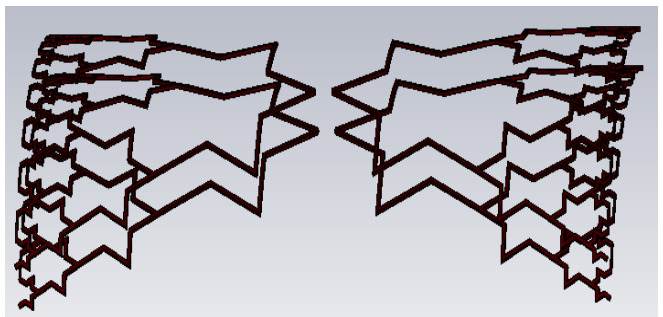

Fig. 12. Fractal array antenna short - bend configuration

\section{Comparison of $\mathbf{S 1 1}$ parameter - array antenna}

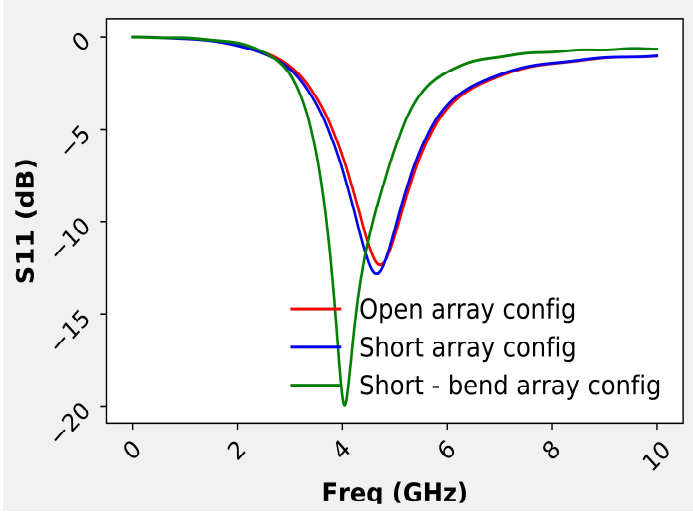

Fig. 13. Comparison of S11 parameter for fractal array antenna

\section{Comparison of gain - array antenna}

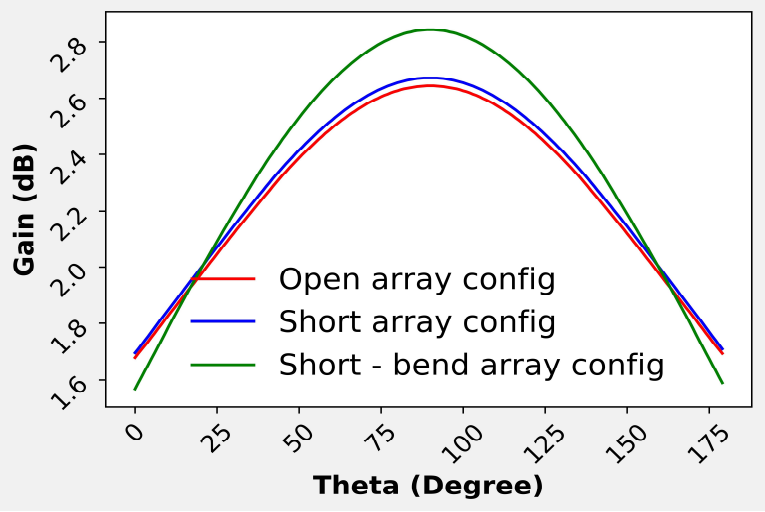

Fig. 14. Comparison of farfield gain for fractal array antenna

\section{Comparison of Impedance - Array antenna}

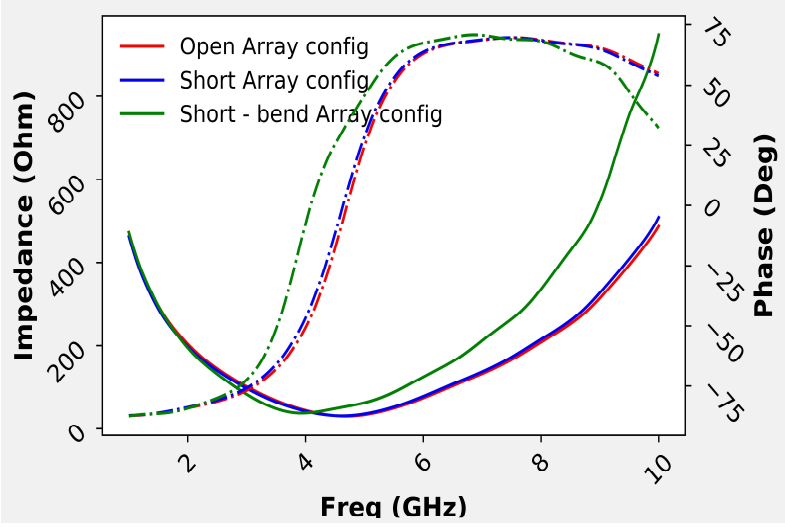

Fig. 15. Comparison of impedance for fractal array antenna

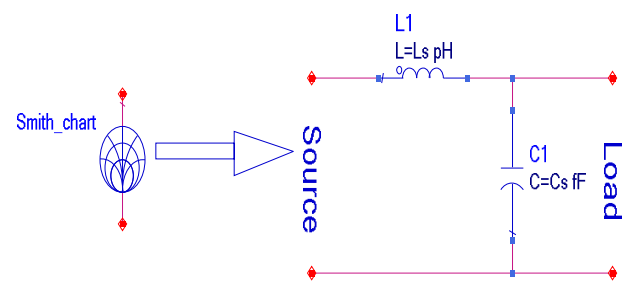

Fig. 16. Impedance matching circuit for fractal array antenna

TABLE II. LC parameters for Impedance matching

\begin{tabular}{|l|c|c|c|}
\hline & $\begin{array}{c}\text { Open config } \\
\text { (array) }\end{array}$ & $\begin{array}{c}\text { Short config } \\
\text { (array) }\end{array}$ & $\begin{array}{c}\text { Short - bend config } \\
\text { (array) }\end{array}$ \\
\hline $\mathrm{L}_{\mathrm{s}}(\mathrm{pH})$ & 782.56733 & 788.131 & 512.10071 \\
\hline $\mathrm{C}_{\mathrm{s}}(\mathrm{fF})$ & 541.75003 & 532.83185 & 491.99835 \\
\hline
\end{tabular}

The solid line presents the gain while the dashed line represents the resonance frequency. As seen here, the array configuration always produces higher gain with a small sacrifice of the dimensional thickness with $0.5 \mathrm{~mm}$ and is consistent across various bending. The farfield gain for single element and array antenna shows a consistent linear behavior for its bend except for the array antenna configuration in the lower bend until $4 \mathrm{~mm}$ while the resonance frequency shows a nonlinear behavior with its bending radius. The array antenna always resonates at lower resonance than the single element.

\section{Peak Gain and resonant frequency with bend}

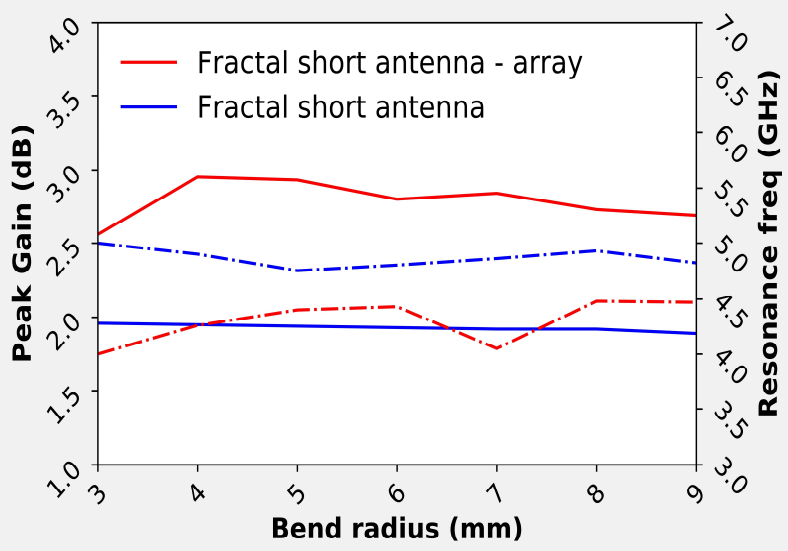

Fig. 17. Gain and Resonant frequency behavior with the change in bend 


\section{Neural network analysis of the farfield gain}

A trained neural network can be used to calculate various parameters from the provided input parameter. In the present case, the gain of the fractal Koch antenna array is calculated with the gain of a single element fractal Koch antenna. The predicted gain of the array for the open end, short end and short end - bend array are obtained and shown in Figs. 18 20. The error plot is also calculated and it shows that the predicted gain is within small error margin of the actual gain calculated with the CST software.

\section{Predicted and actual gain for open antenna}

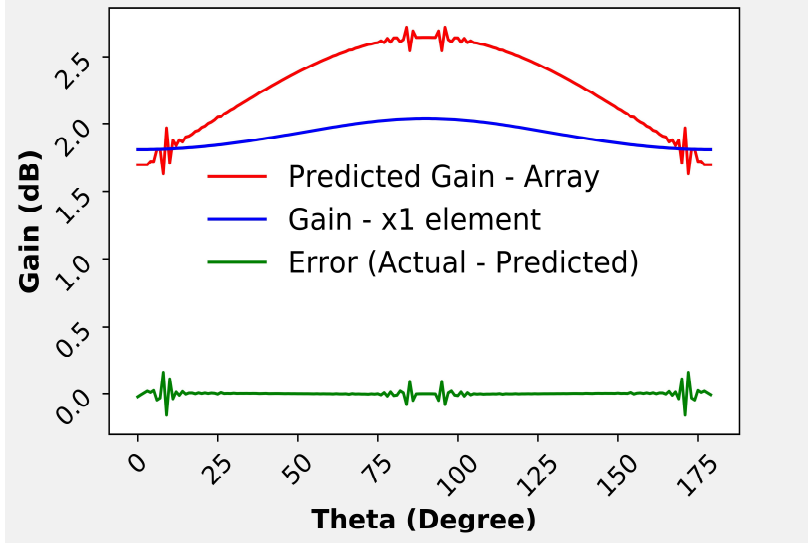

Fig. 18. Predicted gain for open fractal antenna

\section{Predicted and actual gain for short antenna}

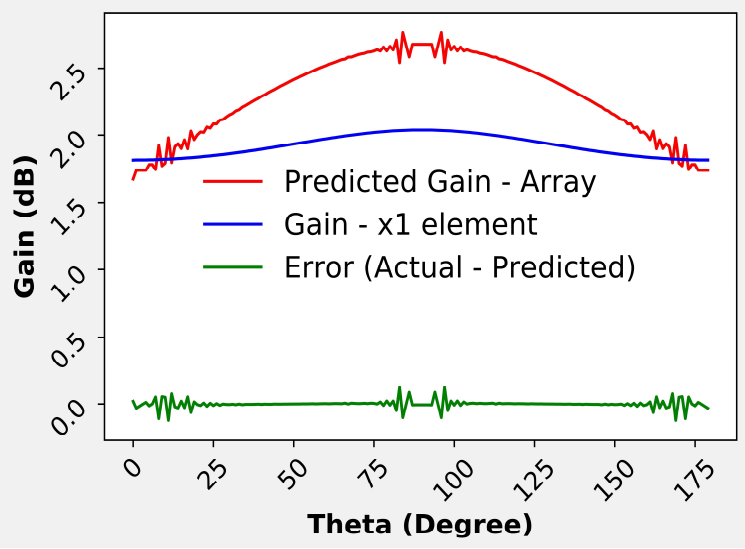

Fig. 19. Predicted gain for short fractal antenna

Predicted and actual gain for short - bend antenna

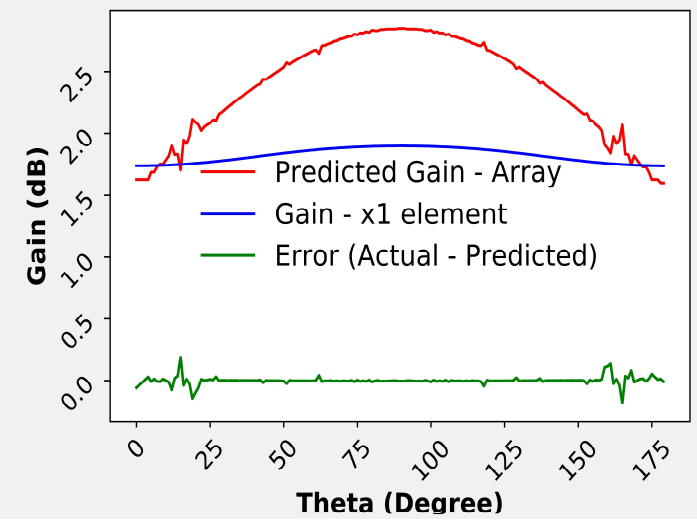

Fig. 20. Predicted gain for short bend fractal antenna

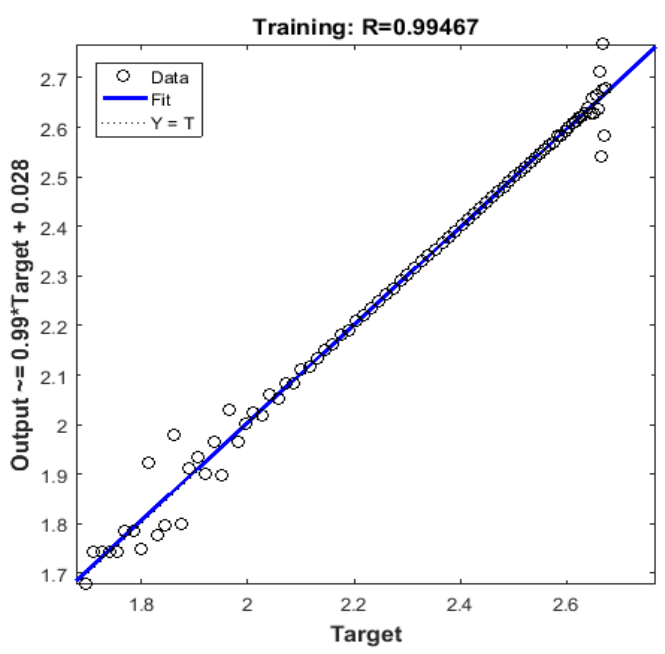

Fig. 21. Plot regression of the gain for short fractal array antenna

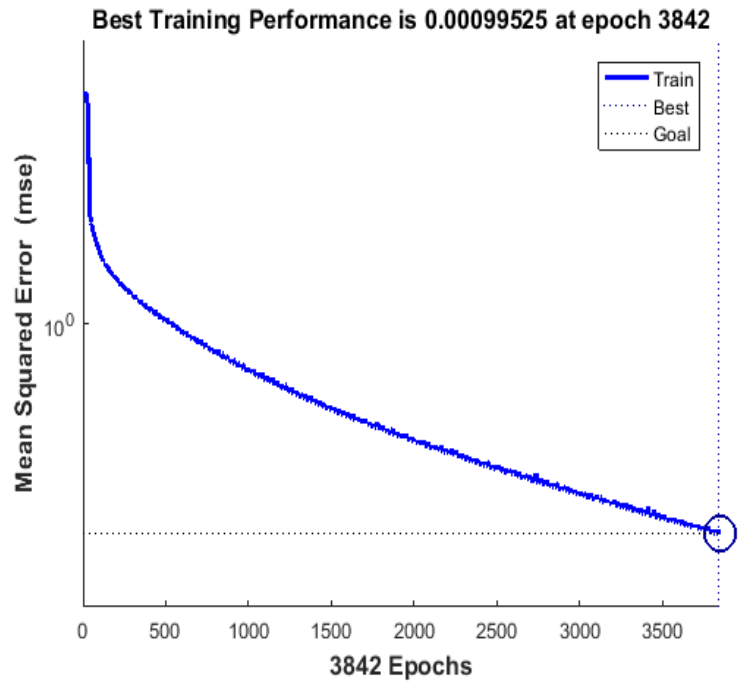

Fig. 22. Plot performance of the gain for short fractal array antenna

The linear plot regression and plot performance are also obtained and shown in Figs $21-22$. The predicted data shows an approximate linear fit to its actual output with 3842 epochs however with an increase in the number of neuron and epoch size, the computational resource can result in perfect linear fit to the desired output. Apart from the gain, other parameters of the antenna such as S11 parameter and directivity show the similar error margin however it is not shown here for its conciseness.

\section{CONCLUSION}

A compact, miniaturized and thin dipole fractal Koch antenna has been designed for wearable and medical applications within $4-5 \mathrm{GHz}$ frequency band. As shown with the parametric plot of the short fractal configuration, a metallic short at its end does not seem to affect the antenna parameters. As shown previously, the array antenna provides a higher gain than its single element however the resonance frequency shifts to the lower side. Using the Smith chart tool, a simple impedance match circuit has been designed to match the antenna impedance within an impedance controlled PCB environment. In addition an increase in the bandwidth has been obtained with the array configuration. Finally an efficient feed forward neural network has been 
designed and trained to calculate the array antenna parameters such as gain with the input of single element antenna gain.

\section{ACKNOWLEDGMENT}

The work was completed during the earlier project at the University of Cambridge and the author would like to acknowledge the Department of Engineering at University of Cambridge for providing the necessary support.

\section{REFERENCES}

[1] B. B. Madelbrot, The Fractal Geometry of Nature, New York: Freeman, 1983.

[2] D. H. Werner and S. Ganguly, "An overview of fractal antenna engineering research," in IEEE Antennas and Propagation Magazine, vol. 45, no. 1, pp. 38-57, Feb. 2003, doi: 10.1109/MAP.2003.1189650

[3] S. R. Best, "A comparison of the resonant properties of small spacefilling fractal antennas," in IEEE Antennas and Wireless Propagation Letters, vol. 2, pp. 197-200, 2003, doi: 10.1109/1-AWP.2003.819680

[4] D. Tumakov; D. Chikrin; P. Kokunin, Miniaturization of a KochType Fractal Antenna for Wi-Fi Applications. Fractal Fract. 2020, 4, 25. doi: $10.3390 /$ fractalfract 4020025

[5] P. Patel, K. Dwibedi, R. Poonkhuzhali, D. Thiripurasundari and Z. C. Alex, "Miniaturized Dipole antenna using Koch fractal technique for wearable application," 2013 International Conference on
Communication and Signal Processing, Melmaruvathur, 2013, pp. 734-737, doi: 10.1109/iccsp.2013.6577153

[6] K. J. Vinoy; J. K. Abraham, V. K. Varadan, Generalized design of multi-resonant dipole antennas using Koch curves. Appl. Comput. Electromagn. Soc. J. 2004, 19, 22-31

[7] S. A. Hamzah, M. K. Raimi, N. Abdullah and M. S. Zainal, "Design, Simulation, Fabrication and Measurement of a 900MHZ Koch Fractal Dipole Antenna," 2006 4th Student Conference on Research and Development, Selangor, 2006, pp. 1-4, doi: 10.1109/SCORED.2006.4339296

[8] D. N. Tumakov, G. V. Abgaryan, D. E. Chickrin; P. A. Kokunin, Modeling of the Koch-type wire dipole. Appl. Math. Model. 2017, 51, 341-360. doi: 10.1016/j.apm.2017.07.007

[9] G. V. Abgaryan, D. N. Tumakov, Relation between base frequency of the Koch-type wire dipole, fractal dimensionality and lacunarity. J. Fundam. Appl. Sci. 2017, 9, 1885-1898. 10.1109/EWDTS50664.2020.9224819

[10] C. P. Baliarda, J. Romeu and A. Cardama, "The Koch monopole: a small fractal antenna," in IEEE Transactions on Antennas and Propagation, vol. 48, no. 11, pp. 1773-1781, Nov. 2000, doi: $10.1109 / 8.900236$

[11] C. M. Bishop, Pattern Recognition and Machine Learning, Springer, 2006.

[12] J. Arif, N. Ray Chaudhuri, S. Ray and B. Chaudhuri, "Online Levenberg-Marquardt algorithm for neural network based estimation and control of power systems," 2009 International Joint Conference on Neural Networks, Atlanta, GA, 2009, pp. 199-206, doi: 10.1109/IJCNN.2009.5179071 